Supporting Information to:

\title{
Electrochemical Behavior of Graphene in a Deep Eutectic Solvent
}

\author{
David Fuchs,,${ }^{\mathrm{a}, \$, \#}$ Bernhard C. Bayer, ${ }^{\mathrm{b}, \mathrm{c}, \#, *}$ Tushar Gupta, ${ }^{\mathrm{b}}$ Gabriel L. Szabo, ${ }^{\mathrm{d}}$ \\ Richard A. Wilhelm, ${ }^{\mathrm{d}}$ Dominik Eder, ${ }^{\mathrm{b}}$ Jannik C. Meyer, ${ }^{\mathrm{c}, \S}$ Sandra Steiner, ${ }^{\mathrm{a}}$ Bernhard Gollas ${ }^{\mathrm{a},{ }^{*}}$
}

anstitute for Chemistry and Technology of Materials, Graz University of Technology, Stremayrgasse 9, A-8010 Graz, Austria

${ }^{\mathrm{b}}$ Institute of Materials Chemistry, Vienna University of Technology (TU Wien), Getreidemarkt 9, A-1060 Vienna, Austria

${ }^{\mathrm{c}}$ Faculty of Physics, University of Vienna, Boltzmanngasse 5, 1090 A-Vienna, Austria

${ }^{\mathrm{d}}$ Institute of Applied Physics, Vienna University of Technology (TU Wien), Wiedner Hauptstraße 8-10, A-1040 Vienna, Austria

\# equal contribution

*corresponding authors:

bernhard.bayer-skoff@tuwien.ac.at, bernhard.gollas@tugraz.at

${ }^{\$}$ Present address: Institute of Energy Technology, University of Duisburg-Essen, Lotharstraße 1, D-47048 Duisburg, Germany

§Present address: Institute for Applied Physics, Eberhard Karls University of Tuebingen, Auf der Morgenstelle 10, D-72076 Tuebingen, Germany and Natural and Medical Sciences Institute at the University of Tuebingen, Markwiesenstr. 55, D-72770, Reutlingen, Germany 


\section{Estimation of internal $i R$ drop in the CVD graphene monolayer}

The internal $i R$ drop of the graphene layer can be estimated from the conductivity of CVD graphene reported as $3 \cdot 10^{6} \mathrm{~S} \cdot \mathrm{m}^{-1} \cdot{ }^{1}$ Applying Ohm's law with a graphene monolayer thickness of $0.4 \cdot 10^{-9} \mathrm{~m}$ (graphene monolayer) and a width of $5 \cdot 10^{-3} \mathrm{~m}$ (as in our electrodes), one can calculate a potential drop of $33.5 \mathrm{mV}$ over a distance of $2 \mathrm{~cm}$ (as in our electrodes) for an initial current of $1 \cdot 10^{-5} \mathrm{~A}$ (as in Figure 4e).

This intrinsic $i R$ drop is small compared to the applied overpotentials during $\mathrm{Zn}$ deposition in Figure 4 and Figure 5. Therefore, a compounding factor to the intrinsic $i R$ drop in pristine graphene is necessary to make the $i R$ drop large enough along the electrode length to result in a macroscopic $\mathrm{Zn}$ deposit gradient, as observed in Figure 4b. We suggest that this factor is the graphene degradation during the sweeps to the cathodic switching potential of $-0.6 \mathrm{~V}$, see main text. 


\section{Supporting Figures}

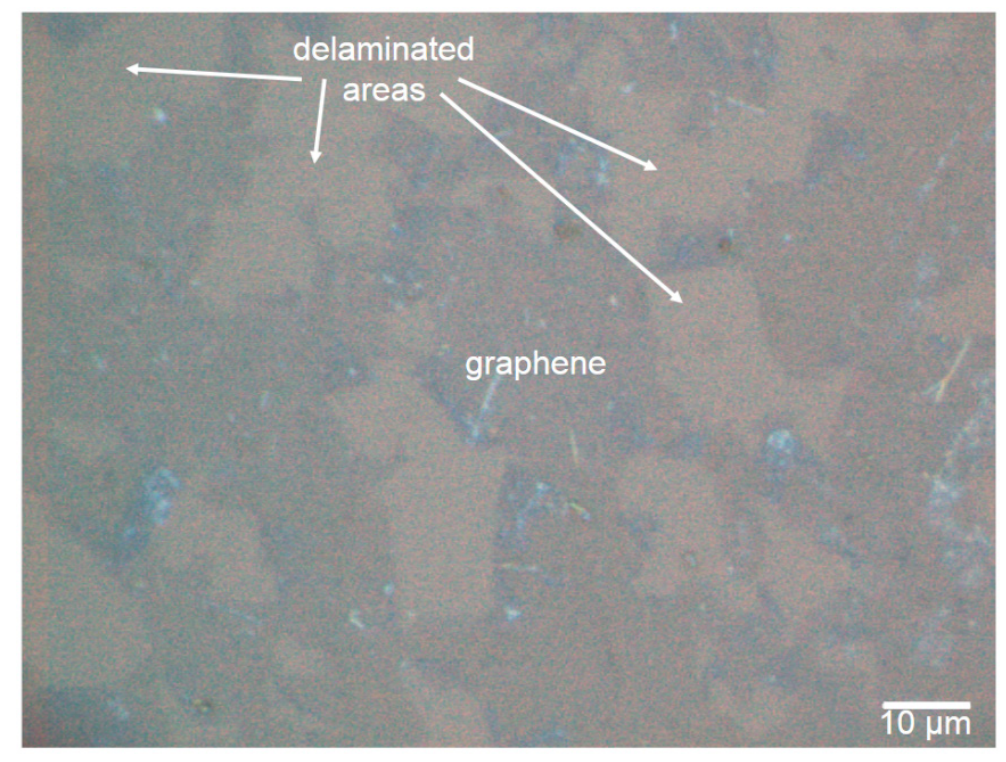

Supporting Figure S1: Optical microscopy of CVD graphene that has been cathodically cycled in 12CE. The image shows clear signs of macroscopic film degradation incl. void formation and delamination. 


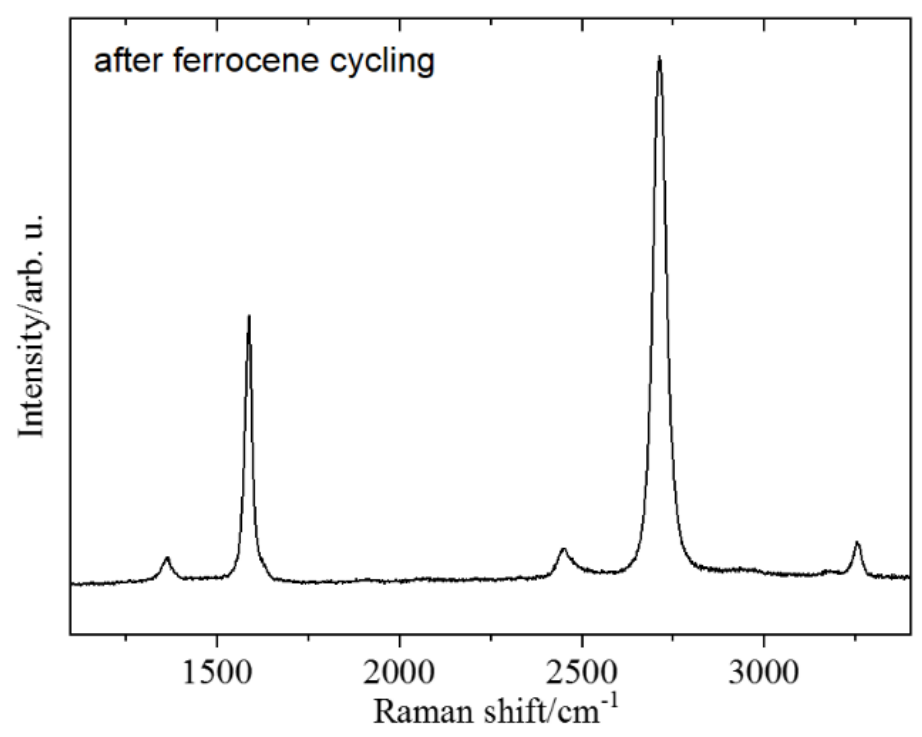

Supporting Figure S2: Raman spectrum of the CVD graphene electrodes after the ferrocene cyclic voltammetry. 


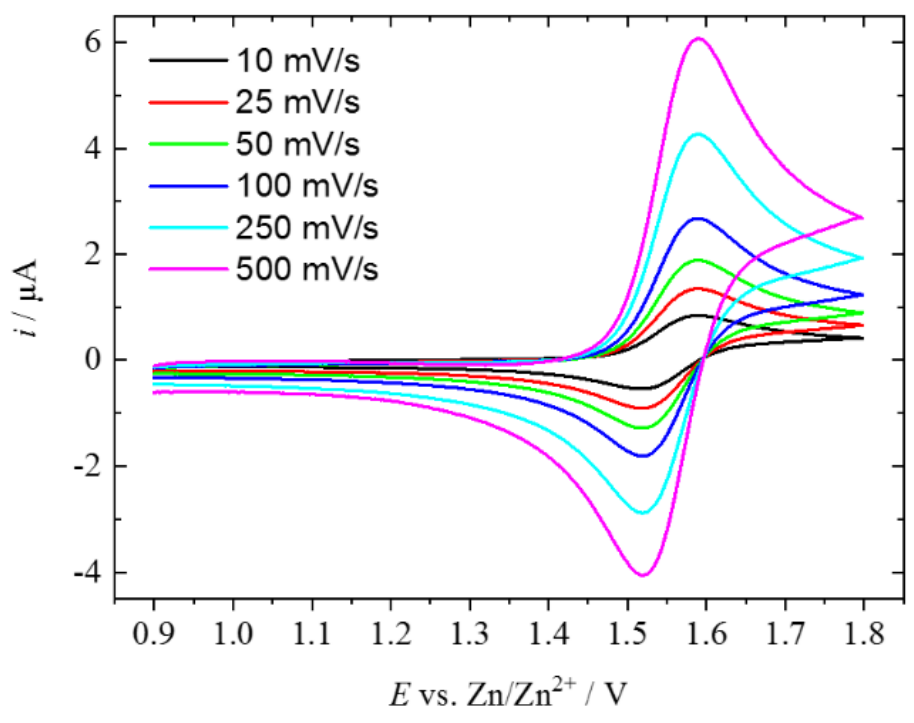

Supporting Figure S3: Cyclic voltammograms of a $1.034 \mathrm{mM}$ solution of ferrocene in $12 \mathrm{CE}$ recorded with a glassy carbon disc ( $3 \mathrm{~mm}$ diameter) working and a Pt mesh counter electrode versus a $\mathrm{Zn} / 0.3 \mathrm{M} \mathrm{Zn}^{2+}$ reference electrode at $25^{\circ} \mathrm{C}$. All curves are background subtracted. For extracted values see Table S1.

Table S1: Cyclic voltammetric peak potentials, peak potential differences, formal potentials and oxidation peak currents in Supporting Figure S3.

\begin{tabular}{lccccc}
\hline $\boldsymbol{v} / \mathbf{V ~ s}^{-\mathbf{1}}$ & $\boldsymbol{E}_{\mathbf{p}}{ }^{\mathbf{0 x}} / \mathbf{V}$ & $\boldsymbol{E}_{\mathrm{p}}{ }^{\text {red }} / \mathbf{V}$ & $\boldsymbol{\Delta} \boldsymbol{E}_{\mathbf{p}} / \mathbf{V}$ & $\boldsymbol{E}^{\mathbf{0}} / \mathbf{V}$ & $\boldsymbol{i}_{\mathbf{p}}{ }^{\mathbf{0 x}} / \boldsymbol{\mu A}$ \\
\hline 0.010 & 1.588 & 1.520 & 0.068 & 1.554 & 0.842 \\
0.025 & 1.590 & 1.517 & 0.073 & 1.554 & 1.348 \\
0.050 & 1.588 & 1.520 & 0.068 & 1.554 & 1.885 \\
0.100 & 1.590 & 1.520 & 0.070 & 1.555 & 2.674 \\
0.250 & 1.588 & 1.520 & 0.068 & 1.554 & 4.269 \\
0.500 & 1.590 & 1.520 & 0.070 & 1.555 & 6.068 \\
\hline
\end{tabular}




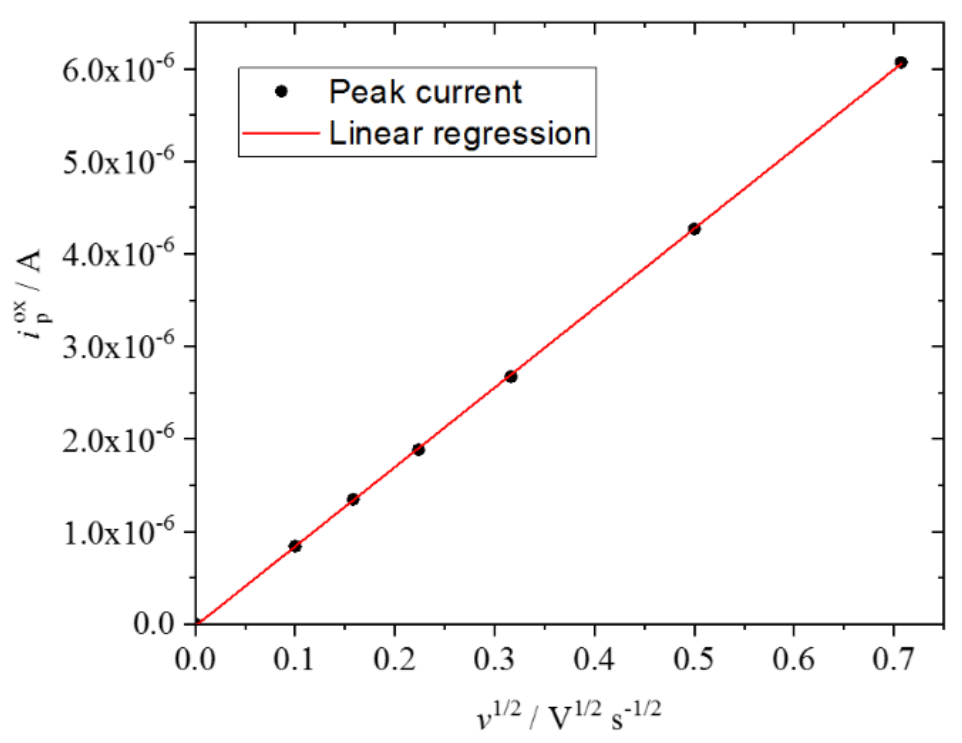

Supporting Figure S4: Background subtracted anodic peak currents (•) of cyclic voltammograms in Supporting Figure S3 plotted versus the square root of the scan rate. The linear regression (-) has a slope of $8.58 \cdot 10^{-6} \mathrm{~A} \cdot \mathrm{s}^{0.5} \cdot \mathrm{V}^{-0.5}$, an intercept of $-1.8 \cdot 10^{-8} \mathrm{~A}$, and shows a Pearson correlation coefficient of 0.99997.

Randles-Sevcik equation: ${ }^{2}$

$$
i_{\mathrm{p}}=0.4463 n F A C\left(\frac{n F v D}{R T}\right)^{\frac{1}{2}}
$$

$i_{\mathrm{p}}$ : cyclic voltammetric peak current

$n$ : number of electrons transferred per molecule

$F$ : Faraday constant

$C$ : bulk concentration of redox active species

$v$ : scan rate

$R$ : universal gas constant

$T$ : absolute temperature 

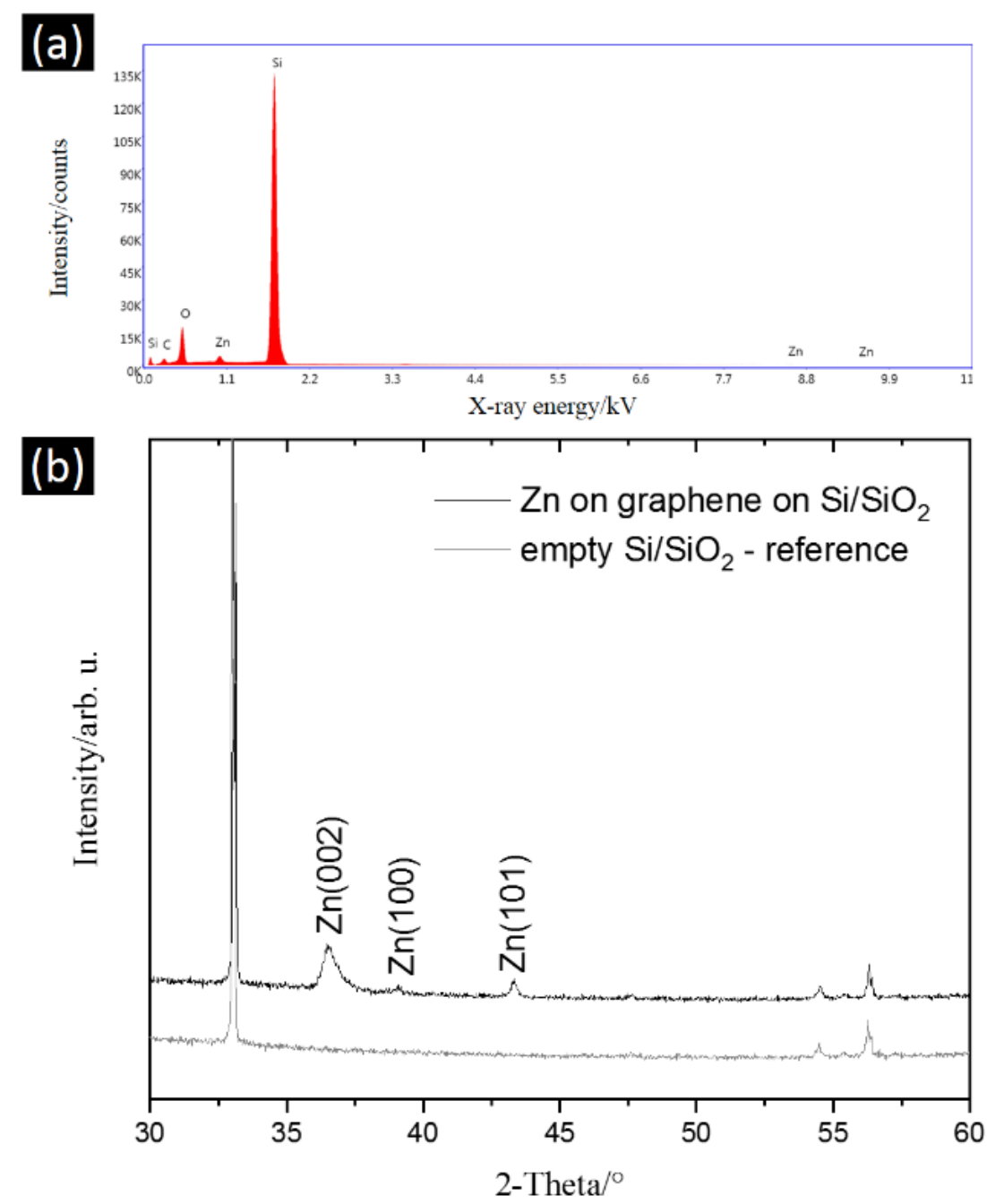

Supporting Figure S5: (a) Energy dispersive X-ray spectroscopy (EDX) of potentiodynamically deposited zinc on graphene on $\mathrm{Si} / \mathrm{SiO}_{2}$ wafer (10 cycles, corresponding to Figure $4 \mathrm{~b}$ and Figure $5 \mathrm{~b}$ ), confirming $\mathrm{Zn}$ deposition via elemental analysis. (b) X-ray diffractometry (XRD) patterns of potentiodynamically deposited zinc on graphene on $\mathrm{Si}_{2} / \mathrm{SiO}_{2}$ wafer (black top line, 10 cycles, corresponding to Figure $4 \mathrm{~b}$ and Figure $5 \mathrm{~b}$ ) and an empty $\mathrm{Si} / \mathrm{SiO}_{2}$ wafer as reference (grey bottom line), confirming that the deposited $\mathrm{Zn}$ is polycrystalline $(\mathrm{P} 63 / \mathrm{mmc}){ }^{3}$ Indexed Miller indices are matched to the International Centre for Diffraction Data (ICDD) file Zn: 01-078-9363. 


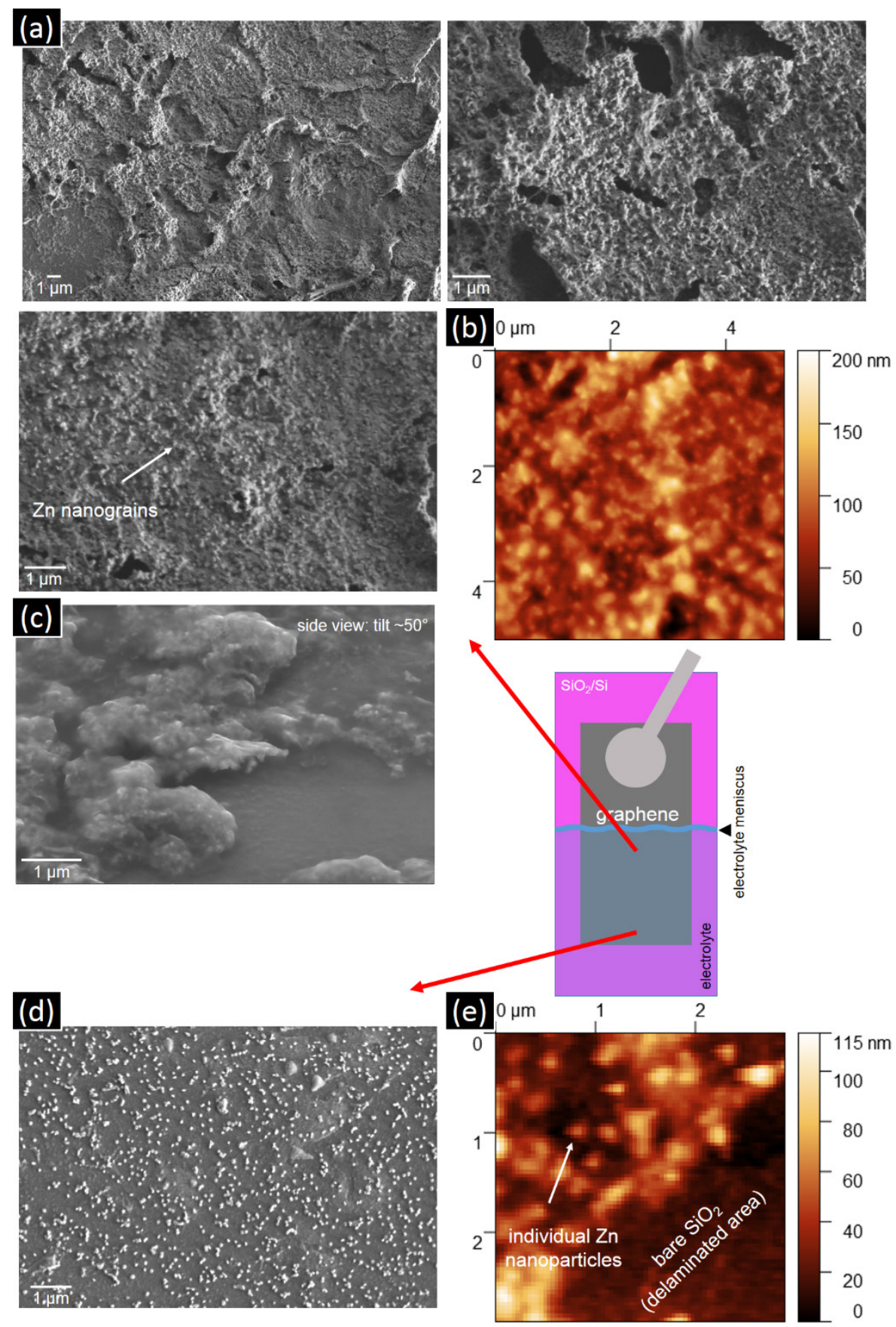

Supporting Figure S6: (a) SEM images at varying magnifications of a graphene electrode with potentiodynamically deposited Zn (10 cycles, corresponding to Figure $4 \mathrm{~b}$ and Figure 5b; first image replotted from Figure $4 \mathrm{~b}$ ) showing an area close to the meniscus of the electrolyte. (b) AFM data corresponding to (a). (c) Side view SEM (tilt $\sim 50^{\circ}$ ) of the $\mathrm{Zn}$ deposits corresponding to (a) to estimate the Zn deposit thickness. (d) SEM image of a graphene electrode with potentiodynamically deposited $\mathrm{Zn}$ (10 cycles, corresponding to Figure $4 \mathrm{~b}$ and Figure 5b; image replotted from Figure $4 b$ ) showing an area at the lower end of the electrode (bottom). (e) AFM data corresponding to (d). 


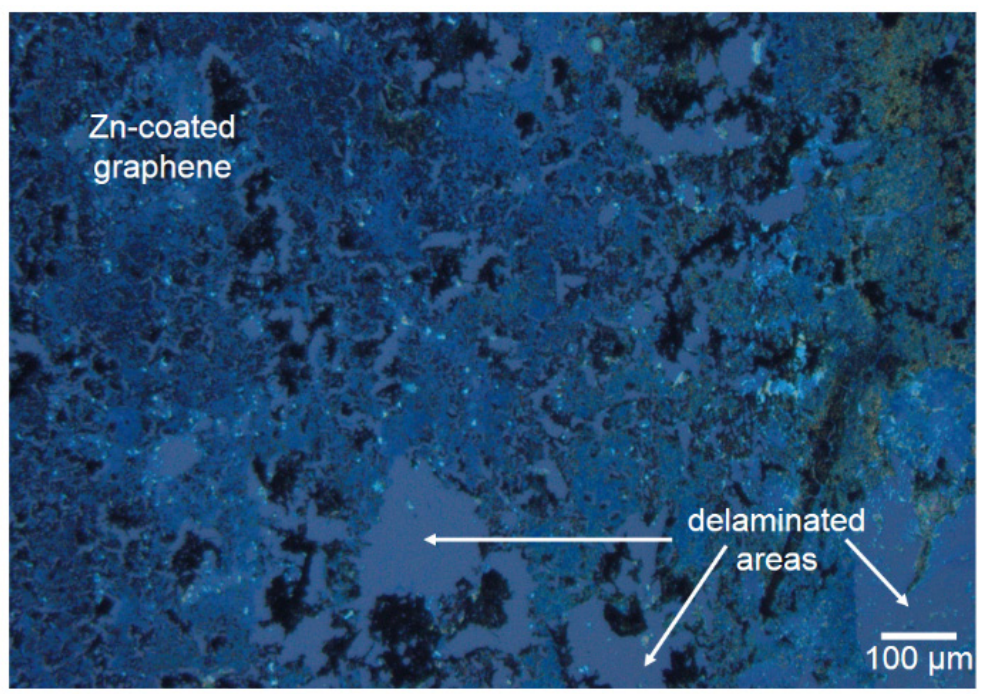

Supporting Figure S7: Optical microscopy of potentiodynamically deposited zinc on graphene on $\mathrm{Si} / \mathrm{SiO}_{2}$ wafer (10 cycles, corresponding to Figure $4 \mathrm{~b}$ and Figure $5 \mathrm{~b}$ ), showing an area at the lower end of the electrode. The image shows clear signs of macroscopic film degradation incl. void formation and delamination. 


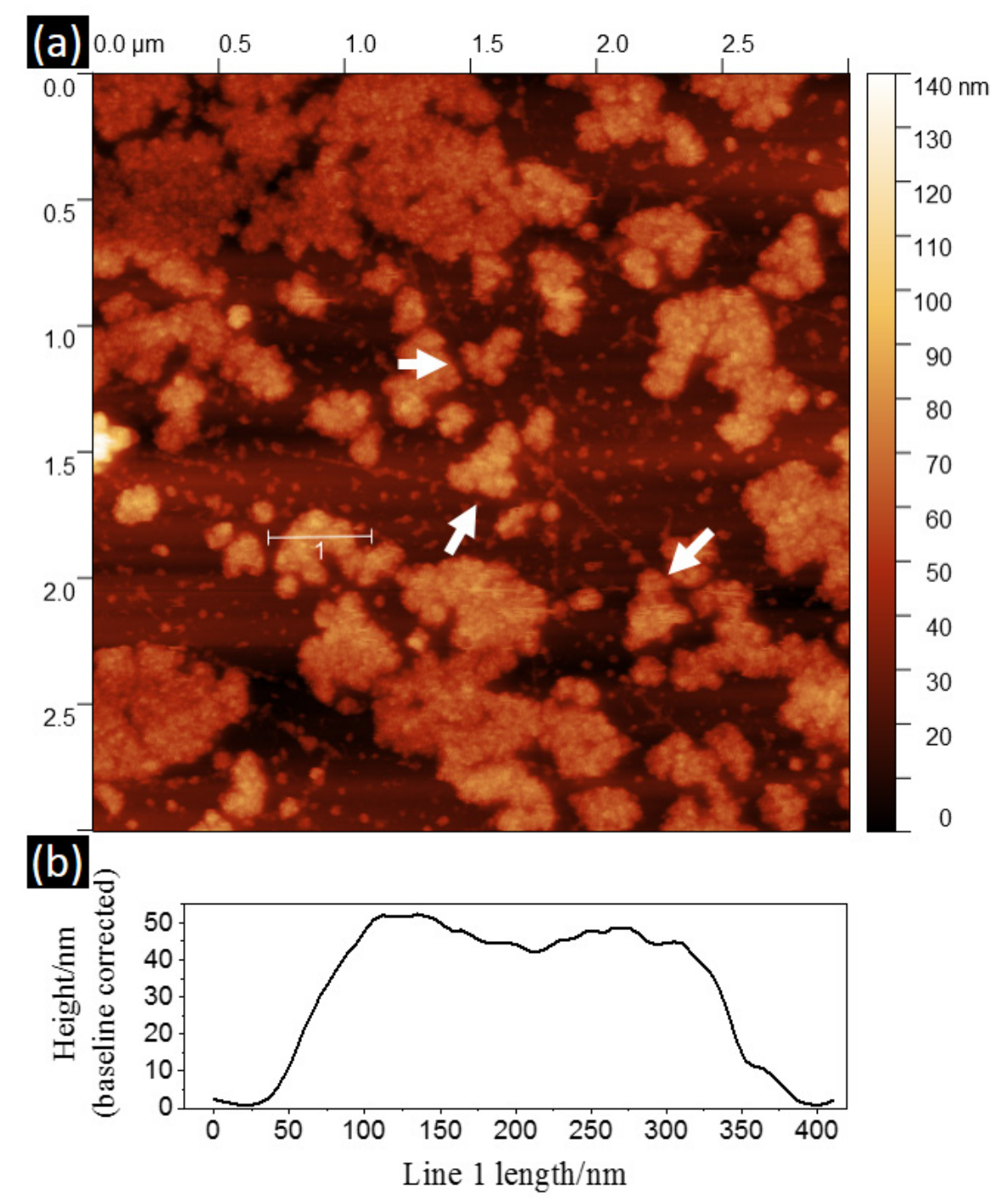

Supporting Figure S8: (a) AFM data of graphene electrode with potentiostatically deposited Ge $(-0.3 \mathrm{~V}$ for $260 \mathrm{~s})$, corresponding to Figure $6 \mathrm{~b}$. White arrows indicate wrinkles in the graphene at which Ge islands nucleated. (b) Baseline corrected AFM height profile trace along the line " 1 " in (a). 

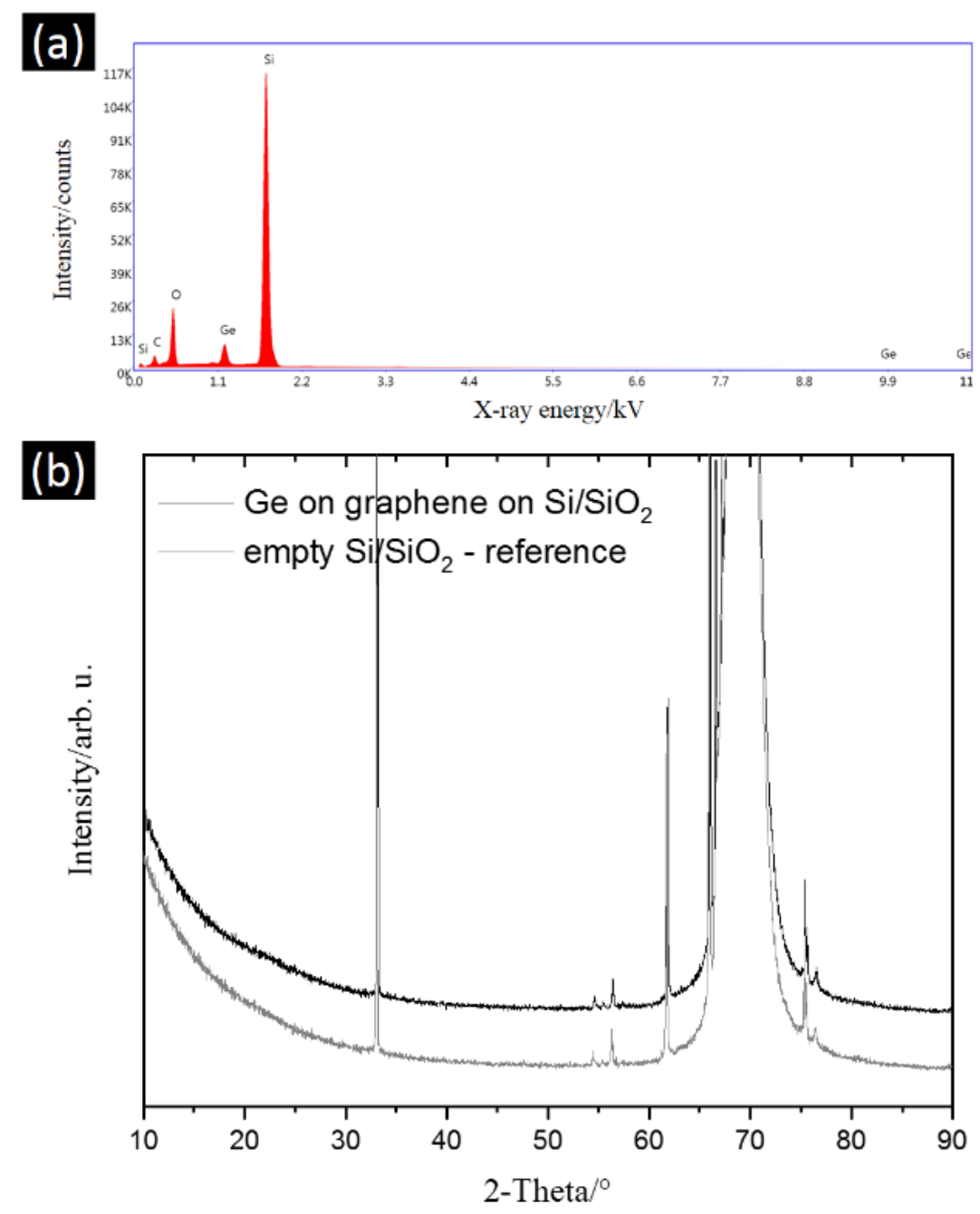

Supporting Figure S9: (a) EDX spectrum of a graphene electrode with potentiostatically deposited Ge ( $-0.3 \mathrm{~V}$ for $7000 \mathrm{~s}$, corresponding to Figure $6 \mathrm{~d})$, confirming Ge deposition via elemental analysis. (b) X-ray diffractometry (XRD) patterns of potentiostatically deposited Ge on graphene on $\mathrm{Si} / \mathrm{SiO}_{2}$ wafer (black top line, $-0.3 \mathrm{~V}$ for $7000 \mathrm{~s}$, corresponding to Figure $6 \mathrm{~d}$ ) and an empty $\mathrm{Si} / \mathrm{SiO}_{2}$ wafer as reference (grey bottom line), whereby the absence of Ge-related reflections indicates that the Ge deposits are amorphous. 


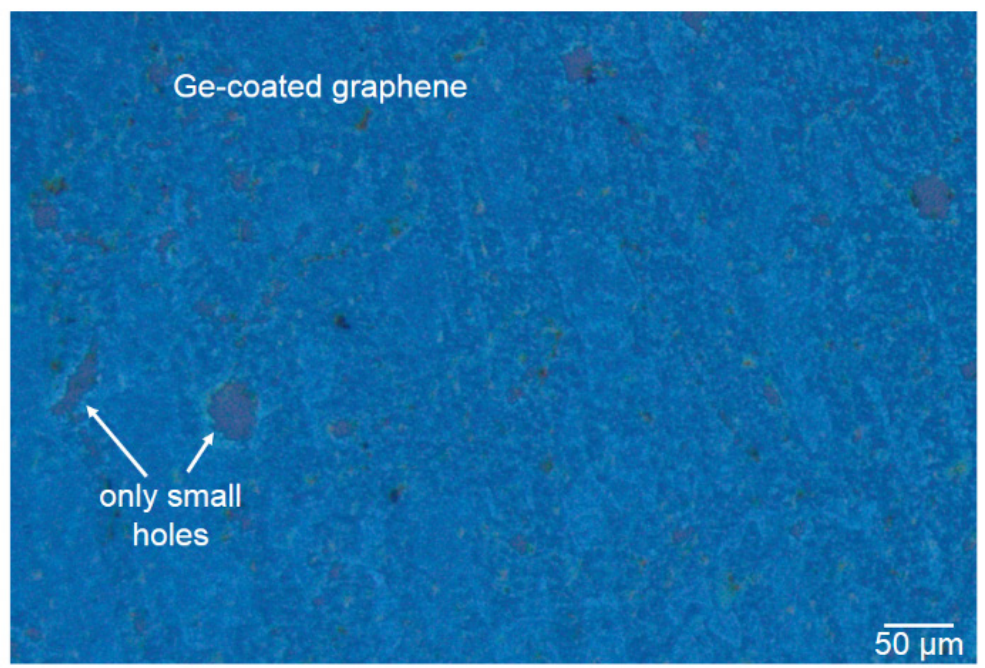

Supporting Figure S10: Optical microscopy of a graphene electrode with potentiostatically deposited Ge ( $-0.3 \mathrm{~V}$ for $7000 \mathrm{~s}$, corresponding to Figure $6 \mathrm{~d})$, showing an area in the center of the electrode. The image shows homogeneous Ge deposition on the graphene and practically no signs of graphene degradation (in particular in comparison to Supporting Figure S7). 


\section{Supporting References}

(1) Yuan, W.; Zhou, Y.; Li, Y.; Li, C.; Peng, H.; Zhang, J.; Liu, Z.; Dai, L.; Shi, G. The Edgeand Basal-Plane-Specific Electrochemistry of a Single-Layer Graphene Sheet. Sci. Rep. 2013, 3, 2248.

(2) Bard, A. J.; Faulkner, L. R. Electrochemical Methods: Fundamentals and Applications, 2nd ed.; Wiley: New York, 2001.

(3) Vieira, L.; Schennach, R.; Gollas, B. The Effect of the Electrode Material on the Electrodeposition of Zinc from Deep Eutectic Solvents. Electrochimica Acta 2016, 197, 344-352. 